\title{
Distributed Fibre-Optic Technology for Security Monitoring of a Structural Load of Road and Motorway Tunnels
}

\author{
David Hruby ${ }^{1}$, Jakub Cubik ${ }^{1}$, Jakub Jaros ${ }^{1}$, Stanislav Kepak ${ }^{1}$, Stanislav Zabka ${ }^{1}$, Pavel Mec ${ }^{2}$, Vladimir \\ Vasinek $^{1}$ \\ ${ }^{1}$ Department of Telecommunications, Faculty of Electrical Engineering and Computer Science, \\ VSB-Technical University of Ostrava \\ ${ }^{2}$ Department of Building Materials and Diagnostics of Structures, Faculty of Civil Engineering, VSB - Technical \\ University of Ostrava
}

\begin{abstract}
The article is focused on the analysis of the use of the distributed fiber optic technology for security monitoring of a structural load of road and motorway tunnels. Brillouin Time Domain Reflectometry (BOTDR) was used to monitor the deformation. The principle is based on the measurement of the stimulated Brillouin scattering. The whole experiment was carried out during the construction of the motorway tunnel in Slovakia. As part of the construction of the tunnel, the standard optical telecommunication fiber was attached to the primary tunnel beams that was subsequently used as a sensor for the BOTDR system. The sensor allows monitoring and analysis of the load on the highway tunnel to ensure the safety of the structural load. In total, there were 6 measurement days, which were scheduled in 5 months of tunnel construction.
\end{abstract}

Keywords: Brillouin Time Domain Reflectometry (BOTDR), Fiber-optic sensor, Tunnel, Sensor.

\section{Introduction}

The construction of tunnels is closely linked to the monitoring of rock movements. At present, standard geodetic methods, string or piezoelectric sensors [1] are used for this geotechnical monitoring. In principle, the monitoring of underground works can be divided into underground monitoring at the construction site itself, and surface monitoring. The objectives of the monitoring are based on basic hypotheses of transformation of the "rock environment - the reinforcement of the underground work" system. By implementing an underground work, the primary state of equilibrium is disturbed, the stress is redistributed, and the concentration of stress behind the stope reaches several times the value of the primary stress. The change in stresses occurs simultaneously with deformations, radial displacements towards the centre of the work arise, and an extrusion (bulging of the face into the stope) may occur. Beyond the work's outline, an area of plastic deformation and abrasion is often formed, which causes an increase in the pressure on the reinforcement of the work. The implementation of an underground construction, especially in the case of so-called shallow tunnelling, is reflected negatively also on the surface - there are sinking basins whose extent and character influence the degree of negative effects of tunnelling on the surface (sedimentation, cracks on objects, disruption of groundwater regime, etc.) [2].

The main objective of the monitoring is to verify the project prerequisites, to apply the so-called observation method of tunnel implementation, when the project is modified on the basis of evaluation of geotechnical monitoring results, to ensure safety during construction and to minimize negative impacts of the construction implementation. The geotechnical monitoring must therefore be an integral part of all tunnelling methods, the essence of which is to achieve an optimal interaction of the rock mass with the reinforcement. Theoretically, the parameters of this optimum interaction of the rock mass with the reinforcement are defined by the working point determined by the Fenner-Pacher curves (work-deformation characteristics of the rock and the reinforcement). An optimum value of a portion of radial rock deformations that occur prior to the reinforcement activation can significantly reduce the load on the reinforcement. However, in case of an inappropriately long delay in the reinforcement activation, a rapid increase in the stope deformation may result in the collapse of the entire excavation [3]. One of the possible ways to extend the classical methods of tunnel load monitoring now is currently offered by optical fibres.

The development of optical fibres causes that current conventional sensors are being slowly but surely replaced by fibre optic sensors. In the current applications for building construction monitoring, one-point Bragg sensors (FBG) or 
distributed sensors based on Brillouin scattering (BOTDR) [4] are being used. Bragg grates are implemented together with anchor rods that are used to strengthen rock bodies. This approach serves to monitor the stress-deformation state in the anchors to warn against rock movements and their disturbances [5]. Further, FBGs are used to measure deformations in the secondary lining of tunnels [6], where quasi-distributed measurements can be used by multiplex techniques [7Systems based on Brillouin scattering are advantageous in terms of distributed distribution of deformations along the entire optical fibre.

For this purpose, special fibre-optic cables with the tight coupling of optical fibres to optical fibre cover materials are used. Current studies show that standard optical fibres [8] can also be used for specific applications. In tunnel construction, distributed BOTDR systems are most commonly used to measure deformations on tunnel lining. In this application, the opening of joints between individual segments of tunnel lining is most often examined in tunnelling the tunnels [9]. Furthermore, these systems are used to monitor the lining of sprayed concrete and rock movements [10]. An advantage of the Brillouin scattering system is that it can cover many times the area of its measurement because the entire fibre used serves as a sensor, unlike the FBG sensor system.

\section{Methods}

\subsection{Brillouin scattering}

A Brillouin scattering gives rise to frequency shifted components and the frequency shift $v_{\mathrm{B}}$ experienced by the Brillouin scattered light is proportional to the acoustic velocity $V_{a}$ of the scattering medium.

Brillouin frequency shift:

$$
v_{B}=\frac{2 n V_{a}}{\lambda_{0}}
$$

Where $n$ is the index of refraction of the fiber and $\lambda_{0}$ the wavelength of the pump. The acoustic velocity $V_{a}$ is directly related to the material density, which is temperature and strain dependent. As a result, the Brillouin frequency shift intrinsically depends on the local temperature and strain. The Brillouin frequency shift therefore is an intrinsic parameter of the silica fiber and its measurement provides information about the local fiber temperature and strain conditions.

Typical temperature and strain dependencies are shown in the hereafter figure [11]:
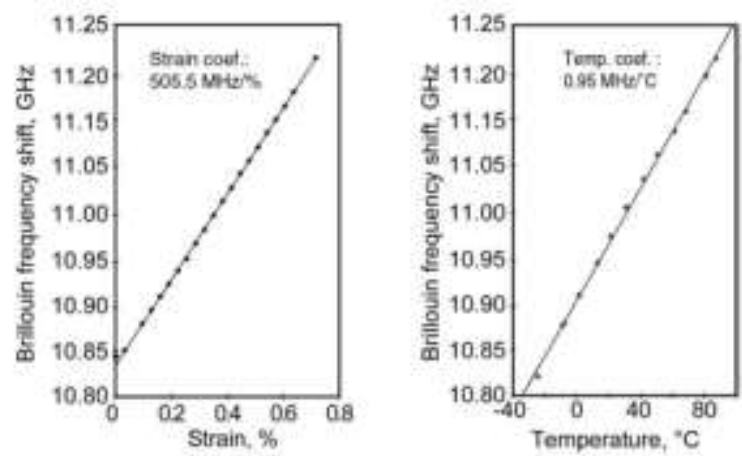

Fig. 1: Typical calibration data of a SMF optical fiber, showing the Brillouin shift temperature and strain dependence at a $1550 \mathrm{~nm}$ wavelength [11].

The Brillouin scattering process has the particularity that it can be stimulated by a second optical signal in addition to the light-wave that generated the scattering, providing that this second signal, called the probe fulfils specific conditions.

This is an especially interesting property for sensing application and can be achieved by the use of a probe beam counter propagating with respect to the pump. Stimulation is maximized when pump and probe frequencies (or 
wavelengths) are exactly separated by the Brillouin shift. In this case, the energy transferred from the pump to the probe results shows a greatly enhanced backscattered intensity and thus a larger Signal-to-Noise Ratio (SNR). This is seen as a resonant phenomenon where an amplification of the probe power occurs at the expense of the pump when the resonant condition is fulfilled, i.e. when the frequency difference between pump and probe matches the local Brillouin frequency. The amplification is proportional to the pump and the probe intensities as well as to the Brillouin gain coefficient. A careful adjustment of the pump/probe levels, and thus of the amplification along the fiber, allows SNR maximization for different optical fibers, measurement conditions, distance range and presence of losses [11].

The temperature/strain information is coded in the Brillouin frequency shift. Scanning the probe frequency with respect to the pump while monitoring the intensity of the backscattered signal allows one to find the Brillouin gain peak, and thus the corresponding Brillouin shift, from which the temperature or the strain can be computed. This is achieved by using a single laser from which both the pump beam (a few nanoseconds long optical pulse) and the probe beam (Continuous Wave - CW light) are created. A telecommunication like optical modulator is used to scan the probe frequency in a controlled manner [11].

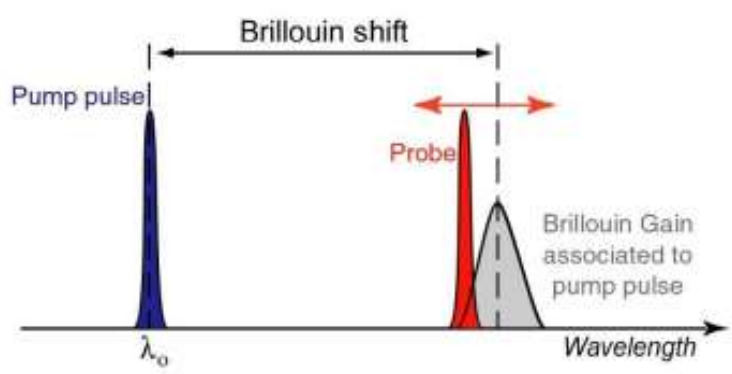

Fig. 2: Brillouin shift [11].

Due to the pulsed nature of the pump, the pump/probe interaction takes place at different location along the fiber at different times. For any given location $\left(l_{1}\right)$, the portion of probe signal which interacted with the pump arrives on the detector after a time delay equal to twice the travelling time from the fiber input to the specified location. Thus, monitoring the backscattered intensity with respect to time, while knowing the speed of light in the fiber, provides information on the position where the scattering took place. The recording of the probe intensity as a function of time at a given frequency is referred to as Frequency Trace or Trace [11].

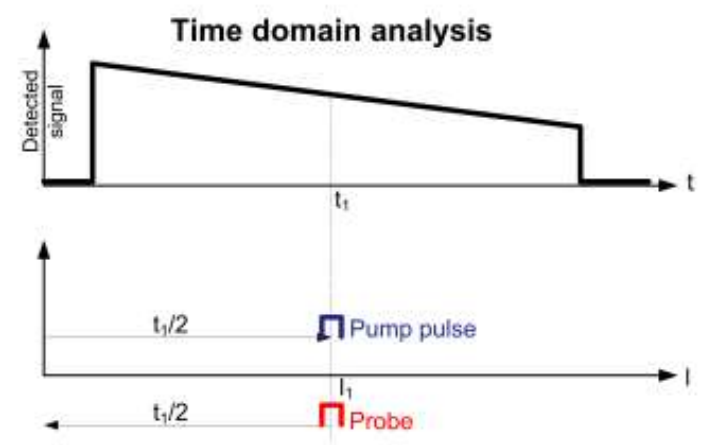

Fig. 3: Time domain analysis [11]. 


\section{Experimental setup}

\subsection{Construction of the tunnel}

The practical part of this article deals with the analysis of the monitoring of highway tunnels using an optical fibre implemented into primary lining. The implementation of the optical cable was carried out during the excavation of a new one-way tunnel in Žilina (Slovak Republic) with a length of $867 \mathrm{~m}$. The area above the tunnel is of an unstable character of agricultural land, forests and meadows with a height of overburden from 5 to $40 \mathrm{~m}$. The highly disturbed mass which consists predominantly of a mixture of clayey and clayey soil induces problems associated with the driving process. The intervention in the stress state of the degraded massif results in a large increase in deformations which stimulate the formation of loose areas around the excavation or tunnel faces. Due to the unstable overburden above the tunnel, Metrostav's primary requirement for the tunnel construction was to monitor the evolution of the load change over time.

The tunnel is driven using the New Austrian Tunnelling Method (NATM), also known as sequential excavation method (SEM). This is a popular method of modern tunnel design and construction.

When this method is used, the whole profile of the tunnel tube is not driven at the same time, the tunnelling ia carried out in individual sections. This makes it possible to use the supporting function of the rock mass itself. An integral part of tunnelling using the new Austrian tunnelling method is geotechnical monitoring, allowing for time optimization when applying tunnel lining.

The lining of the tunnel is composed of two parts, the so-called primary and secondary lining, see Figure 2. The primary lining is implemented immediately after tunnelling works. A lattice or truss beam is installed on the excavation face. This part is reinforced by concrete spraying. The primary lining with a thickness of 200 to $400 \mathrm{~mm}$ then provides temporary stability of the entire excavation. The so-called secondary lining, mostly as a reinforced concrete shell, rarely made of plain concrete, is built as a final lining of the underground work, often using so-called tubing (prefabricated panels). Between the primary and secondary lining, a waterproofing liner is inserted to protect the secondary lining against water for the lifetime of the work.

\subsection{Implementation of optical fiber}

A special optical cable type marked OFS Accudry Flex+ ZWP BIF G.657.A2) was used for the distributed measuring. The optical cable consist of a standard telecommunication single-mode optical fibre in the primary acrylate protection, the secondary protection with a thickness of $900 \mathrm{um}$, a Kevlar thread and an outer protection with a thickness of $3 \mathrm{~mm}$.

The cable has been chosen for its properties suitable for installation or mounting in concrete to measure deformations acting on the given monitored section, as well as for its price that is in the order of euro units per meter. The implementation of the optical cable was carried out on two iron bars of the tunnel truss beam, which, after spraying with concrete, became part of the primary lining. The model of implementation of the optical cable to the iron bars of the truss beam is shown in Fig. 4a, the practical implementation is illustrated in Figure 4b (the cable location highlighted in green). 


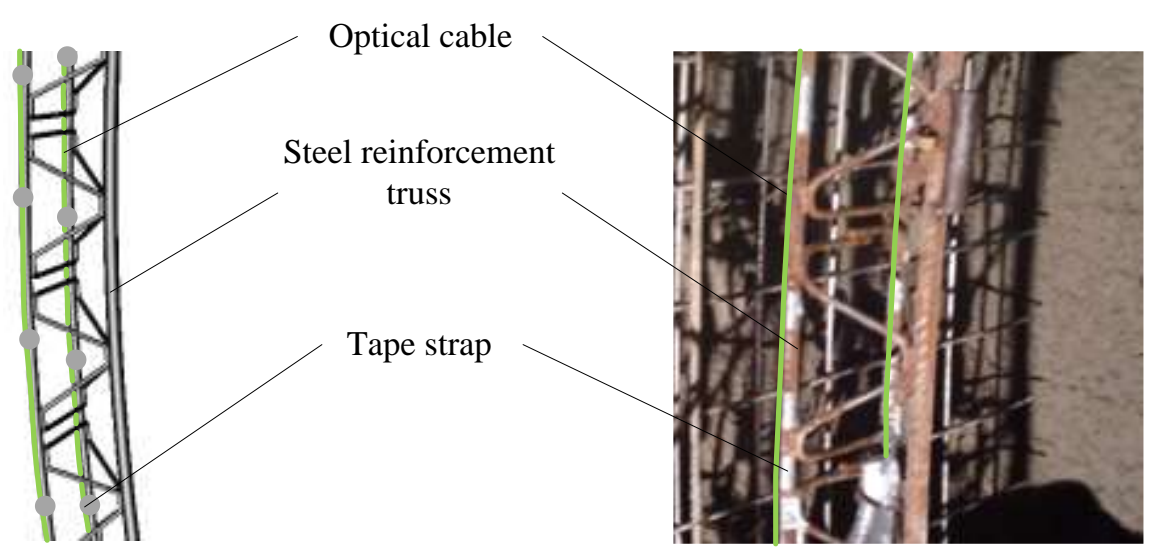

Fig. 4: The implementation of the optical cable on the truss beam of the primary lining (a) schematic illustration; (b) practical implementation.

The primary lining was installed on 14/01/2017 at the distance of $350 \mathrm{~m}$ from the entry to the tunnel. The optical cable was conducted out of the primary lining approximately at a height of $2 \mathrm{~m}$ and deposited to the switchboard cabinet.

\section{Results}

The optical cable was implemented on 14/01/2017 on the truss beam by means of drawn strips. Long-term measurements in the tunnel were conducted on six measuring days over a 5-month time horizon, see Table 1. Individual measurement days were selected depending on the accessibility of the tunnel. During each measurement day, 25 measurements were made for each channel and averaged for subsequent processing. Subsequently, the influence of temperature at Brillouin frequencies was read out based on the temperature measurement by reference temperature sensors installed in the primary lining along the measuring optical cable.

Table 1: Date of measurements.

\begin{tabular}{cc}
\hline ID & Date \\
\hline 1 & $19 / 01 / 2017$ \\
2 & $03 / 02 / 2017$ \\
3 & $22 / 03 / 2017$ \\
4 & $25 / 04 / 2017$ \\
5 & $07 / 06 / 2017$ \\
6 & $21 / 06 / 2017$ \\
\hline
\end{tabular}

Individual averaged measurements from the defined 6 measuring days are shown in Figure 5. 


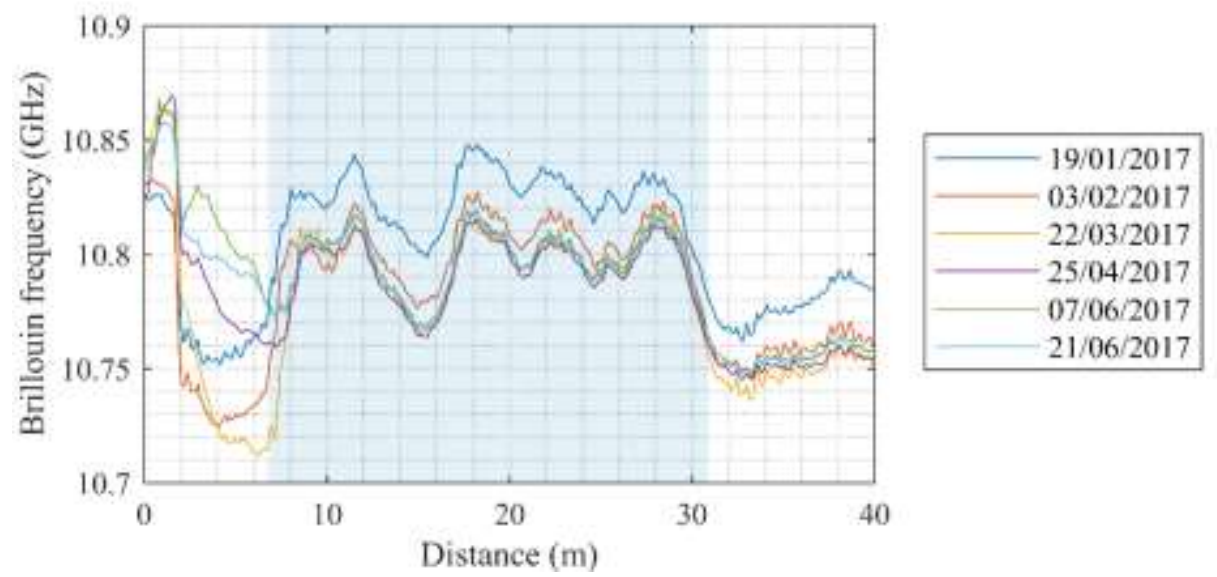

Fig. 5: Courses of the Brillouin frequency from 6 measurements in the course of 5 months according to Table 1.

Individual curves correspond to the absolute value of the Brillouin frequency when measured with the optical cable on six measurement days. The blue area marks the area that corresponds to the length of the optical cable implemented in the primary lining of the tunnel. The waveforms clearly show a drop of the Brillouin frequency out of the area of the cable in the primary lining, which is due to the pre-stressing of the optical cable when it is placed on the truss beams of the primary lining.

The effect of pre-load could be eliminated by performing a reference measurement of the Brillouin frequency prior to spraying the primary lining. This would determine the magnitude of the concrete solidification effect of the primary lining.

Due to the time stress (the succession of Metrostav's work in the excavation did not allow the creation of a time interval for reference measurement) no initial reference measurement with the DSTS was performed before the primary lining was injected. Moreover, because of the unstable overburden of the tunnel, Metrostav's main requirement for the construction of the highway tunnel was to determine changes in the load (changes of the Brillouin frequency) of the tunnel in time, whether pressures on the primary lining increased or the primary lining load was stable. Although reference measurements were not performed, it is possible to track the desired Brillouin frequency changes relative to the first day of measurement. This monitoring has been conducted for the sixth month and will continue until the end of 2017, throughout the construction period. This article analyses data from the first half of 2017.

As for the primary lining load, it is important to know how the load on the monitored part changes during the tunnel construction. Figure 6 shows changes in the Brillouin frequency. Individual curves show a change in the Brillouin frequency relative to the first measurement, which was 4 days after the implementation of the optical cable in the primary lining. 


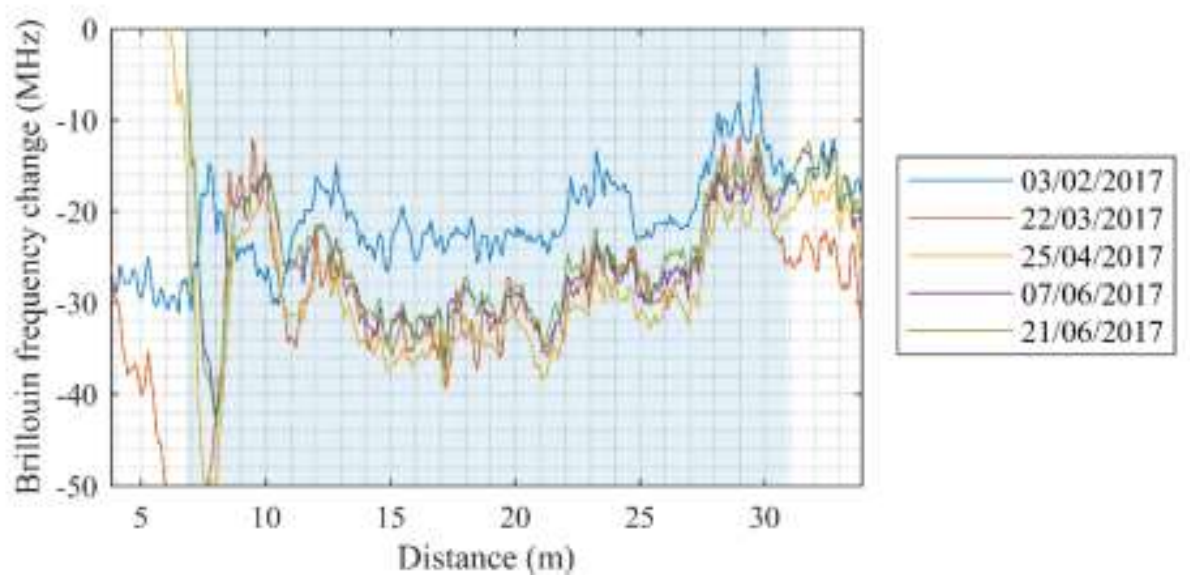

Fig. 6: Changes in the Brillouin frequency from 6 measurements according to Table 1 versus the first measurement of 19/01/2017.

In the first measurement cycle on 03/02/2017, there is a clear decrease in the Brillouin frequency due to the cooling of concrete at elevated temperature due to the concrete hydration $(-20 \mathrm{MHz})$. The following drop may be due to a combination of temperature reduction to the ambient rock temperature and shrinkage of the concrete. Subsequently, initial deformation effects are stabilized. There are no significant changes in the last two measurements, which may indicate relative stillness in the surrounding rock environment, but a considerably long-term character of the rock mass movement has to be taken into account. Further changes in the primary lining load will be monitored in the continued measurement.

\section{Conclusion}

This article describes a using Distributed Fiber Optical System (DSTS) based on the Brillouin Time Domain Reflectometry (BOTDR) for analysis and safety monitoring of the structural loads of road and highway tunnels. The measurement principle is based on the measurement and analysis of the stimulated Brillouin scattering. The article describes the long-term real experimental measurement of tunnel load, which was carried out during the construction of the motorway tunnel in the Slovakia Republic. The measurement horizon was on the orders of several months. The experimental measurements were carried out with a standard optical telecommunication cable with water-absorbing aramid yarns and jacketing of $4.2 \mathrm{~mm}$ diameter.

\section{Acknowledgements}

This article was supported by the Ministry of Education of the Czech Republic (Projects Nos. SP2017/128 and SP2017/79). This research was partially supported by the Ministry of Education, Youth and Sports of the Czech Republic through Grant Project no. CZ. 1.07/2.3.00/20.0217 within the framework of the Operation Programme Education for Competitiveness financed by the European Structural Funds and from the state budget of the Czech Republic. This work was supported by the VŠB-TUO Student grant project SP2019/79. 


\section{References}

[1] P. Lunardi, Design and Construction of Tunnels: Analysis of controlled deformation in rocks and soils (ADECO$R S)$. Milano: Springer, 2008.

[2] S. Argyroudis, A. M. Kaynia, and K. Pitilakis, "Development of Fragility Functions for Geotechnical Constructions: Application to Cantilever Retaining Walls," Soil Dynamics and Earthquake Engineering, vol. 50, pp. 106-116, 2013.

[3] M. Al-Mukhtar, S. Khattab, and J.-F. Alcover, "Microstructure and Geotechnical Properties of Lime-Treated Expansive Clayey Soil," Engineering Geology, 2012.

[4] G. Song, W. Li, B. Wang, and S. C. M. Ho, "A review of rock bolt monitoring using smart sensors," MDPI AG, vol. 17, no. 4, 2017.

[5] T. M. Do and Y. S. Kim, "Prediction of load transfer depth for cost-effective design of ground anchors using FBG sensors embedded tendon and numerical analysis," Geomechanics and Engineering, vol. 10, no. 6, pp. 737-755, 2016.

[6] S. Li, J. Ma, and J. Hu, "Rockfall hazard alarm strategy based on FBG smart passive net structure," Photonic Sensors, vol. 15, no. 1, 2015.

[7] M. Fajkus, I. Navruz, S. Kepak, A. Davidson, P. Siska, J. Cubik, and V. Vasinek, "Capacity of wavelength and time division multiplexing for quasi-distributed measurement using fiber bragg gratings," Advances in Electrical and Electronic Engineering, vol. 13, no. 5, 2015.

[8] M. Fajkus et al., "Effect of the geometric deformations on the Brillouin scattering in the standard single-mode optical fiber," Proc. SPIE 9889, 2016.

[9] F. Wang, H. Huang, D. Zhang, W. Zhang, and R. Xu, "Deformation sensing method of shield tunnel based on optical fiber sensing technology of BOTDA," Chinese Journal of Rock Mechanics and Engineering, vol. 32, no. 9, pp. 1901-1908, 2013.

[10] R. A. Moffat, J. F. Beltran, and R. Herrera, "Applications of BOTDR fiber optics to the monitoring of underground structures," Geomechanics and Engineering, vol. 9, no. 3, pp. 397-414, 2015.

[11] OMNISENS, "User Manual DITEST STA-RTM - Fiber Optic Distributed Temperature and Strain Analyzer". Omnisens SA, 3 Rjond Bosson, CH-1110, Morges, Switzerland. 